\title{
FE-CALCULATIONS OF STRESS DISTRIBUTION UNDER PRISMATIC AND CONICAL SANDPILES WITHIN HYPOPLASTICITY
}

\author{
J. Tejchman ${ }^{1}$ and $W . W u^{2}$ \\ ${ }^{1}$ Gdańsk University of Technology, \\ Gdańsk, Poland \\ ${ }^{2}$ Institut für Geotechnik, Universität für Bodenkultur, \\ Vienna, Austria
}

\section{Introduction}

The static and dynamic behaviour of sandpiles has attracted much attention. Simple experiments with prismatic and conical piles of granular materials indicate, contrary to intuition that the maximum vertical normal stress does not always appear directly beneath the pile vertex but at a certain distance from the apex. It was found later that the occurrence of the stress dip at the heap centre strongly depends on the method of pile construction [1]. In the case of the raining procedure by means of a sieve located above the heap, the pressure maximum occurs at the centre of the sandpile. However, when a funnel procedure (centric flow out of a hopper) is used, a pressure peak is obtained away from the centre, where a significant pressure dip appears. The pressure dip is usually more pronounced in conical heaps than in prismatic ones.

In the present paper, the stress distribution under sandpiles is studied with the FEM and micro-hypoplasticity without imposing any additional condition [2]. The analyses were performed with a micro-polar hypoplastic model which is suitable to investigate the phenomenon of the granular heap construction since it takes into account the effect of the direction of deformation rate. The calculations were carried out with prismatic and conical heaps composed mainly of an initially dense cohesionless sand.

\section{Micro-polar hypoplastic model}

Granular materials consist of grains in contact and surrounding voids. Thus, their micromechanical behaviour is inherently discontinuous, heterogeneous and non-linear. Despite their discrete nature, the mechanical behaviour of granular materials can be reasonably described by the principles of continuum mechanics. Non-polar hypoplastic constitutive models have been developed at Karlsruhe University, where the stress rate tensor is assured to depend on the stress tensor, strain rate tensor and the void ratio via isotropic non-linear tensorial functions based on the representation theorem. The constitutive models were formulated by a heuristic process considering the essential mechanical properties of granular materials undergoing homogeneous deformation. A striking feature of hypoplasticity is that the constitutive equation is incrementally nonlinear in deformation rate. The hypoplastic models are capable of describing some salient properties of granular materials, e.g. nonlinear stress-strain relationship, dilatant and contractant volumetric change, stress level dependence, density dependence and strain softening. A further feature of hypoplastic models is the inclusion of the critical states, i.e. states in which a grain aggregate can deform continuously at constant stress and volume (void ratio). In contrast to elasto-plastic models, a decomposition of deformation into elastic and plastic parts, the formulation of a yield surface, plastic potential, flow rule and hardening rule are not needed. The hypoplastic models were initially proposed for cohesionless soil. The hallmarks of these models are their simple formulation and procedure for determining material parameters with standard laboratory experiments. The material parameters can be related to the granulometric properties of granular materials, such as grain size distribution curve, shape, angularity and hardness of grains. A further advantage lies in the fact that one single set of material parameters is valid for a wide range of pressures and densities. Hypoplastic constitutive models without a characteristic 
length cannot describe the scale effects associated with shear bands such as thickness and spacing of shear bands. A characteristic length can be introduced into hypoplasticity by means of the micropolar, non-local and second-gradient theory. In this paper, a micro-polar theory is adopted [3], [4]. A micro-polar model makes use of rotations and couple stresses, which have clear physical meaning for granular materials.

\section{FE-results}

The analyses were carried out for a plane strain case and an axi-symmetric case. In the calculations, the symmetry axis was assumed. The pile was discretized with 200 triangular elements. The heap inclination to the bottom was assumed to be $\alpha=30^{\circ}$, which was equal to the critical internal friction angle of sand. The size of elements was not larger than $5 \times d_{50}$, which was sufficient to obtain meshindependent numerical results.

The construction of the heap was simulated in 10 stages using two different methods, viz. the raining procedure and the funnel procedure. The sandpile was subject only to gravitational load in the vertical direction. The effect of the following parameters was investigated: a) construction method, b) mean grain diameter, c) base roughness, d) heap inclination and d) initial void ratio of sand.

\section{Conclusions}

A micro-polar hypoplastic constitutive model was used to study the stress distribution der the sandpile. The vertical stress distribution was dependent on the method of the heap construction, The stress increases monotonically up to the apex of the sandpile for the raining procedure. In turn, the stress showed a maximum beyond the heap mid-point for the funnel procedure. The stress distribution did not depend upon the initial void ratio, mean grain diameter, heap inclination and base roughness. The results were similar for prismatic and conical sandpiles.

The FE-results confirm the experimental results by Vanel et al. [1] and numerical results by Al Hattamleh et al. [5]. However, in contrast to the numerical results by Hattamleh et al. [5], no any additional condition (as orientation of initial slip lines) was imposed. The non-uniform distribution of the vertical normal stress beneath the sandpile during a funnel procedure was a natural numerical outcome.

\section{References}

[1] L. Vanel, D. Howell, D. Clarck, R.P. Behringer and E. Clement (1999). Memories in sand: experimental test of construction history on stress distribution under sandpiles. Physical Review E, 60, 5, R5040-R5043.

[2] J. Tejchman and W. Wu (2008). FE-calculations of stress distribution under prismatic and conical sandpiles within hypoplasticity. Granular Matter (accepted for publication).

[3] J. Tejchman and A. Niemunis (2006). FE-studies on shear localization in an anisotropic micropolar hypoplastic granular material, Granular Matter, 8, 3-4, 205-220.

[4] J. Tejchman and W. Wu (2007). Modeling of textural anisotropy in granular materials with stochastic micro-polar hypoplasticity. International Journal of Non-Linear Mechanics 42, 882-894.

[5] O. Al Hattamleh, B. Muhunthan and H.M. Zbib (2005). Stress distribution in granular heaps using multi-slip formulation. Int. Journal for Numerical and Analytical Methods in Geomechanics, 29, 713-727. 